\title{
Communication Médiatisée par Ordinateur: le "lien social" entre juxtaposition, assemblage et hybridisme
}

\section{Theophilos Rifiotis ${ }^{1}$}

${ }^{1}$ Universidade Federal de Santa Catarina, Florianópolis, SC, Brasil 


\section{Résumé}

Il s'agit d'un essai critique sur la notion de "communication médiatisée par ordinateur" écrit à partir de l'auto-ethnographie d'une expérience personnelle. La description de cette expérience permet de mettre en avant une série de problèmes théoriques dans le domaine de la "cyberculture". La réflexion présentée ici a comme but de produire une problématisation des présupposés implicites dans la notion de "communication médiatisée par ordinateur" et de discuter les itérations avec l'ordinateur en mettant en perspective la notion de "lien social". L'essai est dialogue avec les questions clés du champ de l'étude de la technique et de l'ethnographie dans le domaine de la "cyberculture". Les questions qui émergent de cet essai sont celles de l'agentivité sociotechnique, de l'assemblage et de l'hybridisme dans l'univers des rapports "humains-ordinateurs".

Mots-clés: Cyberculture. Agentivité. Hybridisme. Lien Social.

\section{Resumo}

Trata-se de um ensaio crítico sobre a noção de "comunicação mediada por computador", escrito a partir de uma autoetnografia de uma experiência pessoal. A descrição da experiência permite colocar em primeiro plano uma série de problemas teóricos no campo da "cibercultura". $A$ reflexão apresentada aqui tem como objetivo produzir uma problematização dos pressupostos implícitos na noção de "comunicação mediada por computador" e discutir as interaçóes entre o computador, colocando em perspectiva a noção de "laço social". O ensaio é um diálogo com as questóes-chave dos estudos da técnica e da etnografia no campo da "cibercultura". As questões que emergem deste ensaio são as de agência sociotécnica, reagregação e hibridismo no universo das relações "humanos-computadores".

Palavras-chave: Cibercultura. Agência. Hibridismo. Laço Social. 


\title{
1 Introduction
}

\begin{abstract}
Penses-y bien: lorsqu'on t'offre une montre, on t'offre un petit enfer fleuri, une chaîne de roses, une geôle d'air. On ne t'offre pas seulement la montre, joyeux anniversaire, nous espérons qu'elle te fera de l'usage, c'est une bonne marque, suisse à ancre à rubis, on ne t'offre pas seulement ce minuscule picvert que tu attacheras à ton poignet et promèneras avec toi. On t'offre - on l'ignore, le plus terrible c'est qu'on l'ignore -, on t'offre un nouveau morceau fragile et précaire de toi-même, une chose qui est toi, mais qui n'est pas ton corps, qu'il te faut attacher à ton corps par son bracelet comme un petit bras désespéré agrippé à ton poignet. On t'offre la nécessité de la remonter tous les jours, l'obligation de la remonter pour qu'elle continue à être une montre ; on t'offre l'obsession de vérifier l'heure aux vitrines des bijoutiers, aux annonces de la radio, à l'horloge parlante. On t'offre la peur de la perdre, de te la faire voler, de la laisser tomber et de la casser. On t'offre sa marque, et l'assurance que c'est une marque meilleure que les autres, on t'offre la tentation de comparer ta montre aux autres montres. On ne t'offre pas une montre, c'est toi le cadeau, c'est toi qu'on offre pour l'anniversaire de la montre. (J. CORTAZAR)
\end{abstract}

\footnotetext{
Q uand j'ai pris connaissance de la Chaire Singleton (2017), tout de suite l'expression "lien social" m'a interpelé et plus encore par la définition de l'Axe 2 qui s'approchait des questions sur lesquelles je me suis dédié durant mes études du numérique: “documenter différentes formes de transferts/relations" et "explorer des relations entre protagonistes humains et non humains". Alors, j'ai voulu saisir l'occasion pour partager quelques résultats des recherches que nous menons au GrupCiber (Groupe de Recherche en Anthropologie du Cyberspace) au Brésil depuis 1996.
} 
Cependant, le développement du texte final de la communication a connu un important renversement à cause d'un incident qui l'a complément bouleversé. Cet incident m'a fait reconsidérer mon projet d'exposé et m'a poussé à le refaire au complet. Il m’a aussi entrainé dans un processus qui m'a fait retrouver une callosité au bout des doigts de ma main droite que j'avais oublié et que j'ai vite redécouvert au contacte prolongé avec mon stylo plume. Je vais vous expliquer mon pari dans cette nouvelle version de mon exposé.

\section{Un Incident comme les Autres}

Voilà que mon Mac, Macbook Air, compagnon inséparable depuis quatre ans, ma mémoire vive, mes fiches, mes notes..., mon bon à tout m'a "soudainement" lâché. Et du coup, je me suis retrouvé face à un étrange objet (j'ai failli dire "chose"), à la fois bien connu, intime et insolite. Cela m'était arrivé il y a maintenant exactement dix-neuf jours... Le plus important sur le moment c'était le fait que j'avais à peine une matinée avant un rendez-vous Skype avec un jury de thèse au Brésil. La veille, j'avais éteint mon ordinateur après avoir fini mon rapport d'évaluation de cette thèse; et le lendemain matin, je ne parvenais plus à le faire démarrer... Ou plus exactement, comme je l'avais expliqué par téléphone à l'Assistance Technique d'Apple: "Il ne démarre pas!", [...] "il me donne un écran noir", [...] "mais il n'est pas mort, l'écran montre un fond noir avec des petits traits blancs en mouvement, comme les aiguilles de seconde d'une montre". (Le langage courant est tout à fait clair sur mon rapport ambigu avec l'objet, particulièrement, vis-à-vis de son agentivité (agency), puisque jusqu'à la veille nous ne faisions qu'un dans le travail d'écriture, et soudainement il a pris le relais sur moi et mon travail.)

Vous pouvez bien deviner mon désarroi... Eh oui! Je n'avais pas de backup sur ma clé USB, ni sur le nuage ou sur mon compte Dropbox et moins encore une copie papier. Évidemment, j'y avais pensé, mais..., mais... la fatigue..., il était tard et j'étais épuisé, et en plus, lui, mon Mac, était toujours si fiable!!! Il faut le dire et le répéter: sans doute c'était de l'imprévoyance. Mon intention ici c'est de mettre en avant exactement cette négligence et surtout la "surprise", "l'étonnement" 
que j'avais éprouvés face à un fait si banal et prévisible ou tout au moins d'un risque. Je me rends compte maintenant que je me suis laissé glisser de la "confiance" à l'invisibilité de la complexité de la machine ("mon Mac"), et c'est bien autour de cette réflexion que se situe ce texte.

L'incident en question fut comme un rappel de la médiation que l'ordinateur fait avec mes textes, mes idées. Au fur et à mesure que mes liens se renforçaient avec l'ordinateur - un dispositif avec lequel $\mathrm{j}$ 'entretiens une relation chaque fois plus quotidienne et automatique - , un effacement est en cours. Ou dans les termes de M. Akrich, c'est une liaison, un réseau entre moi et la machine qui se produit et dont l'expérience concrète est bien représentée par l'idée de la "boîte noire":

La transformation de faits sociotechniques à des faits tout court passe donc par la transformation de l'objet technique en boîte noire: il s'efface dans le même temps qu'il est plus indispensable que jamais. (AKRICH, 2010 (orig. 1987), p. 217)

Plus qu'avoir incorporé la machine, au sens qu'elle et moi nous ne faisions qu'un, c'est une transformation qui est en cours: l'objet technique devient une boîte noire. Comme nous le savons bien, la notion de "boîte noire" est centrale pour l'anthropologie des sciences (cf. Bruno Latour, "La science en action", 2006). Mais ici, il s'agit de penser les liens entre "humain" et "non humain" et les modes concrets par lesquels ces liens deviennent une possibilité sociotechnique, c'està-dire un réseau qui fait émerger des agents, des éléments qui ont de l'agentivité, qui font faire ${ }^{1}$. D'où la distinction entre objet technique et monde social devient tellement floue qu'elle perd son sens. C'est ainsi que je peux dire qu'avec mon Mac je voyais mes idées sur l'écran, mon texte prenait vie, sans que je pense au clavier (que je venais forcement de toucher) ou au logiciel, au disque dur, etc., etc...

Je reviens à mon "petit" incident, pour rappeler que sur le coup de la mauvaise "surprise", j'avais appelé l'assistance technique, mais que le "problème" n'a pas pu être réglé par téléphone. Autrefois, un "problème" similaire a été réparé lorsque le/a technicien/enne avait 
pris la commande de mon Mac par le réseau internet. Cela n'a pas été possible cette fois-ci.

La terreur du coup a donné place à une issue, un véritable soulagement, quand je reprends mon portable, mon smartphone, à la main. De telle sorte que, quelques heures avant mon rendez-vous Skype, j'ai pu avoir accès au fichier PDF de la thèse et ainsi refaire un guide avec mes commentaires et mes questions pour le doctorat et bien sûr participer à la soutenance. Mon téléphone mobile, mon portable (mon GSM, comme on dit en Belgique) confirme alors son caractère d" "objet fétiche". Pendant mon séjour à Paris il n'est plus tout à fait un téléphone, mais un moyen d'avoir accès aux personnes très chères par WhatsApp, mon guide GPS, RATP, mais aussi mon agenda, calendrier, agence bancaire, outil de prévision du temps, accès aux courriels, appareil photo, réveil, etc. Bref, dans un sens tragique: "il m'a sauvé"... Ou, heureusement j'avais mon portable et le réseau (et la batterie et...). Le portable nous ouvre tout un champ de recherche sur ce qu'on pourrait appeler les technossocialités contemporaines.

En résumé, disons seulement pour le développement de mon argument que la question du "lien" avec les objets ou médiatisé par les objets, pour moi, ne va pas dans le sens d'une explication des comportements "accros" ou d'une "nomophobie" (no-mobilephone-phobia), ou de la "bulle" individualiste, ni vers la société d'information et d'autres notions générales. À mon sens, ces notions nous imposent des contextes explicatifs donnés d'avance, imposant des limites dans lesquelles l'ethnographe devrait identifier des agents et les organiser (Voir Marilyn Strathern "Fora de contexto: as ficções persuasivas da antropologia", "Hors contexte: les fictions persuasives de l'anthropologie", 2010). Comme j'essaie d'argumenter par la suite, le contexte analytique préalablement construit a pour effet de se transformer en cause externe aux observations de terrain, pour leur donner un sens, tout en annulant les médiations concrètement observées. $C^{\prime}$ est dans et par la description que je crois que nous pourrions mieux saisir l'expérience des liens sociotechniques, c'est-à-dire médiatisés² 


\section{Quelques Conséquences du Récit de l'incident}

Dans la petite histoire que je raconte ici, il est donc question de prendre au sérieux la "surprise" face à l'incident et, par conséquent, mon attachement à l'ordinateur. Mon attention se concentre donc sur les situations concrètes observées sur le terrain, particulièrement les attachements entre sujets ("humains") et dispositifs sociotechniques. Il est alors question de réfléchir ici sur un abordage qui en soi-même définit une place spécifique pour la recherche du numérique: la description des attachements, les séries de connexions, tel que l'avait envisagé Bruno Latour à partir de la notion d'attachement de Antoine Henion au Centre de Sociologie de l'Innovation (CSI) à l'École des Mines de Paris. Dans un texte intitulé "Vous avez dit attachements?", Antoine Hennion cherche a nous montrer cette approche de la notion d'attachement:

Qu'est-ce qui nous attache, à quoi est-on attaché ? La question a un autre mérite, qui découle des caractères précédents, mais qui faisait moins ressortir sa première formulation, 'à quoi tient-on ?', un peu coup-de-poing : elle n'est pas seulement, comme on vient de le saisir pas à pas, à la fois indéterminée, contraignante, et pourtant étroitement liée aux situations, elle est aussi symétrique. Est-ce nous qui tenons à quelque chose, ou ce quelque chose qui nous tient ?... Au-delà d'une telle réciprocité, qui resterait très dualiste, les liens de natures très diverses dans lesquels nous sommes pris, qui nous tiennent et qui nous font tenir, appellent une redistribution de l'agence, déployée dans cet entrelacs où chaque lien fait quelque chose, mais où aucun ne suffit. Comme Bruno Latour le relevait, l'idée d'attachement implique aussitôt une remise en cause de la causalité, au profit d'interactions moins nettes : des poussées, des frottements, des entraînements réciproques. Plus de partage clair entre des choses déterminantes et des choses déterminées, on passe de l'oscillation binaire active/passive à la continuité, moins tranchée, mais autrement productive, d'une action distribuée, un 'faire faire' disséminé dans ces réseaux [...]. (HENNION, 2010, p. 181) 
C'est bien le caractère relationnel et contingent de l'attachement et l'abordage sans un centre d'action ni un "acteur" privilégié (qui serait couramment un "humain"), ce qui nous intéresse ici. Il s'agit d'un lien en quelque sorte symétrique qui est au cœur de l'action où nous voulons trouver un langage pour le décrire. Il s'agit de la description des liens entre les "humains" et "non humains" (encore une fois sans en faire une nouvelle dichotomie), et par cette description identifier les configurations de réseau qui en découlent. Le réseau serait aussi une image très appropriée pour décrire les liens, les associations, les attachements, les relations entre "humains" et/ou entre "humains" et "non humains"3.

Dans les grandes lignes, un tel projet se propose de faire un registre des "banalités quotidiennes" et de la transformation des objets "utilisés" et/ou "intéressants" à titre divers, qui deviennent peu à peu "nécessaires" (parfois vitaux) et, finalement, "disparaissent", et, à la limite se confondent avec les corps et les sujets eux-mêmes. C'est ce mouvement qui me fascine et pousse mon intérêt de recherche vers l'approche sociotechnique et à la critique de la notion de "lien" et ses rapports avec la notion de "réseau"4.

L'approche que j'essaie de dessiner ici est redevable des débats contemporains issus des ethnographies mélanésiennes (particulièrement celles de Marilyn Strathern) et amérindiennes (Eduardo Viveiros de Castro), mais aussi des études des sciences (Michel Callon et Bruno Latour). C'est une approche sociotechnique dont l'importance s'impose de plus en plus par la présence massive et croissante des objets techniques et son ubiquité dans la vie quotidienne. Après tout, dans une autre approche, comme le disait Jean Jamin (2004, p. 9) “Un objet n'est pas la simple expression ou concrétion d'une relation; son existence matérielle pas plus que sa fonction ne préjugent de sa destinée culturelle".

Ou encore: "En somme, l'objet ne se réduit pas à la chose, celle-ci n'étant jamais que la totalité virtuelle de ses 'objectivations' concurrentes ou successives." (JAMIN, 2004, p. 9)

Selon Madeleine Akrich (2010, p. 49),

Le sociologue des techniques se trouve devant un objet qui, bien que clairement défini dans son aspect physique, 
n'en est pas moins curieusement insaisissable: les objets techniques se donnent d'emblée comme composites, hétérogènes; mi-chair, mi-poisson, on ne sait par quel bout les prendre. Ils renvoient toujours à une fin, une utilisation pour laquelle ils sont conçus, en même temps qu'ils ne sont qu'un terme intermédiaire sur une longue chaîne qui associe hommes, produits, outils, machines, monnaies $[\ldots]$.

C'est exactement sur le caractère "composite" que nous éprouvons un manque des ressources analytiques (théoriques, méthodologiques, mais aussi politiques et éthiques), malgré les efforts dans le domaine du numérique, spécialement les travaux de Christine Hine (2000; 2005), pour faire face à l'ampleur et l'encroûtement des dispositifs techniques dans la construction de nos expériences quotidiennes, et plus directement sur la place des objets dans la recherche anthropologique.

Le nœud analytique pourrait se trouver dans la façon de concevoir les réseaux, puisqu'il implique couramment l'adjectivation implicite de "réseaux sociaux" dont les points d'interaction sont les individus et/ou les institutions, avec une invisibilisation des médiateurs techniques, par exemple. Sans aller plus loin, la révision que nous envisageons comme projet futur pour ce débat nous rapproche d'un profond débat sur la notion même de relation, qui serait, pour M. Strathern (2010, p. 271), "la substance de l'empirisme anthropologique" à partir duquel nous menons nos recherches. La notion de relation qui nous paraît le mieux représenter cette approche serait celle qui pourrait s'appliquer à n'importe quelle forme de connexion avec tous les éléments qui participent au cours de l'action observée et à décrire. Dans le champ d'études du numérique, les relations sont toujours médiatisées par des éléments techniques qui peuvent passer d'intermédiaire à médiateur au cours de l'action, et donc leur agentivité n'est pas une propriété fixe, mais au contraire, toujours performative.

C'est cette approche performative qui me semble permettre le mieux la rupture avec le débat entre une vision apocalyptique (surveillance, contrôle, individualisme, etc.) et son opposé, la perspective apologétique (accès à l'information, moyen de suppuration des contraintes locales, outil de transparence, démocratisation, universalisation de production 
de contenu, etc.). C'est ainsi que notre groupe de recherche a travaillé pour sortir, si je peux me permettre, d'une espèce de "choix de Sophie" entre le caractère disruptif du web (VIRILO, 1993) et l'intelligence collective (LEVY, 2003). Un jeu sans issue entre deux pôles, tous les deux possibles et adéquats à des contextes particuliers, dont la "solution" était de changer les termes du débat en mettant en avant l'observation et le récit ethnographique suivant le cours de l'action observée. Mais nous sommes bien conscients que dans ce domaine il reste beaucoup de travail à faire et les défis ne sont qu'à peine esquissés.

De telle sorte qu'une simple interrogation de départ paraît exiger une révision profonde de nos moyens d'enquête. Comment décrire, disons, "ethnographiquement" les expériences des sujets avec leurs portables? En fait, juste pour donner un extrait d'un tel projet de recherche, je voudrais rappeler un scénario courant dans nos voyages aériens. À chaque voyage aérien, je me rends compte de l'impatience avec laquelle les passagers (et parfois moi-même) éteignent leurs portables. L'industrie a beaucoup investi pour en finir avec la déconnexion dans les airs. La solution la plus courante demeure "le mode avion" [...] L'atterrissage reste un moment de soulagement, chaque fois moins problématique, mais le soulagement, disons, vient de la possibilité d'allumer son portable. Faut-il encore rappeler les préparatifs du voyage? Le chargeur... Ou avant : le choix de l'appareil, moment où se mêlent facilement des éléments économiques, les marques (qualité et statuts), logiciels, processeur, stockage, etc... Le smartphone est un objet fétiche, véritable joujou... Un objet qui n'est point réductible à sa matérialité et externalité vis-à-vis des sujets (comme le témoignent les portables qui probablement sont à côté de chacun en cet exact moment).

Alors, les questions de fond pour la recherche se sont multipliées: comment produire un récit dans lequel nous puissions faire état des connexions (voire attachements) entre objets, logiciels, réseau wifi, etc., et où s'y mêlent, si je peux ainsi m'exprimer, les sujets humains, leurs valeurs, leurs pratiques, besoins, désirs, etc.? Un univers plein de croisements et d'hybridisation, un carrefour où se passe concrètement l'action, dans lequel il nous faut suivre les éléments en assemblage au cours des interactions. 
Les travaux de Madeleine Akrich dans ce domaine sont exemplaires. Elle montre dans ces études du numérique comment peuvent se croiser les intérêts et les pratiques de santé avec les éléments techniques d'informatique. Dans son article "Comment décrire un objet technique", elle nous propose une façon de procéder qui correspond à ce que j'appelle notre volonté de savoir sociotechnique. Une approche qui essaie de rendre compte de la "force des choses", la "puissance" des objets à devenir des "interprètes du fait social", notre "boîte à conserve", pour reprendre ici les expressions de Marcel Mauss5.

Ne blâmons ni ne louons, il y a d'autres choses dans la vie collective que les techniques, mais la prédominance de telle ou telle technique dans tel ou tel âge de l'humanité, qualifie les nations. (MAUSS, 2004, p. 438).

En résumé, je pense que ces questions ne sont pas exactement nouvelles, mais il faut revenir sur l'œuvre de Marcel Mauss, par exemple, ainsi qu'à la "théorie acteur-réseau" de Michel Callon et Bruno Latour, pour repenser la place des objets dans les analyses du numérique. Certes, les écrits de Marcel Mauss en témoignent déjà des premiers jalons, comme les années 1980 ont été riches et témoignent aussi des avancés faites dans le domaine de la sociologie des sciences. De telle façon que je crois que le moment est venu de systématiser ces acquis, sans oublier bien d'autres comme le témoigne la Revue Technique et Culture, et, dans notre domaine particulier, tous les essais ethnographiques du numérique.

Concrètement, pour ce qu'il en est de nos expériences de recherches au Brésil au GrupCiber, sans aucune prétention d'exemplarité ni de nouveauté, puisqu'il s'agit de problèmes posés déjà à la fin des années '80 et début '90 (RIFIOTIS, 2012), je me permets d'exposer un bref bilan des démarches et des aléas de nos travaux ${ }^{6}$. S'agissant d'un collectif de jeunes chercheurs, quelques-uns actuellement professeurs, et dont l'esprit ouvert m'a beaucoup stimulé à être toujours très critique, nous avons pu élaborer un bilan collectivement de nos travaux. Cette évaluation globale de plus de 20 ans de recherches dans le domaine du numérique m'a permis de travailler l'idée des enjeux de nos politiques 
ethnographiques, qui est d'ailleurs le titre de notre dernier ouvrage collectif (SEGATA; RIFIOTIS, 2016).

Les politiques ethnographiques sont une problématisation des choix qui s'imposent à chaque instant sur le terrain et l'écriture ethnographique: identifier les "acteurs", ou plus précisément les agents (ceux qui font faire), les hybridismes, la distribution de l'agentivité (dans une perspective de symétrisation entre les humains et non humains), ainsi que les modes de faire nos récits pour produire cet effet chez les lecteurs.

\section{Quelques Points de Repère d'un Parcours de Recherche}

Nos premières études en 1995 portaient sur les échanges d'e-mails dans une liste de discussion. Nous avons alors décrit les règles des échanges, le style oral de l'écriture, les flames (disputes internes dans la liste), les off-topics (postages considérés non conformes au but de la liste), les négociations identitaires (qui étaient des moments de grande intensité dans les échanges). Nous avons pu identifier la distribution asymétrique de la prise de parole dans la liste, les connexions hors liste (IRC et ICQ) ou même des appels téléphoniques entre les participants, "complémentaires" aux échanges de la liste (ce qui représentait un défi de réfléchir sur les connexions online et offline, mais aussi les canaux alternatifs dans le web: l'IRC ou l'ICQ, l'usage en parallèle à la liste courante à l'époque. Le logiciel était en quelque sorte l'outil qui définit la modalité de communication. L'analyse détaillée des logiciels nous a permis de problématiser la notion capitale (j'ai failli dire "omnipresente") de l'ethnographie du virtuel et de la netnographie: la communication médiatisée par ordinateur $(\mathrm{CMO})^{7}$.

Nos travaux nous ont montré le rôle médiateur des logiciels: comment ses outils à la fois transportent les informations, permettent le contact, mais aussi les transforment ou modulent les échanges. Nous avons identifié et décrit, par exemple, ce que nous avons appelé l'automatisme du replay, les couches de participation. Concrètement, avec l'accent sur les logiciels, nous avons constaté que la plus grande partie des études ne faisaient qu'une approche de communication 
"intermédiée" par ordinateur, au sens que la technique (le logiciel en l'occurrence) est plutôt un élément neutre. Un des résultats majeurs de prendre au sérieux le caractère médiateur des logiciels a été que nos descriptions nous ont aussi confirmé les limites des recherches sur les numériques fondées sur des notions comme "appropriation", "usage", "signification". Ces trois approches impliquent une extériorité de l'objet par rapport au sujet (humain) et préserve une séparation radicale entre sujet et objet qui nous approche d'un dualisme que nous voudrons problématiser (RIFIOTIS, 2012).

Depuis la fin des années 1990 jusqu'à 2007, nos recherches ont suivi les changements constants et chaque fois plus accélérés de notre terrain de recherche et nous ont obligés à investir sur des multiples plateformes: The Palace, les web-blogs et l'Orkut (en quelque sorte une préhistoire du Facebook). Nous avons alors testé les limites de nos premières expériences sociotechniques dans l'étude de différentes plateformes. Les principes analytiques généraux étant les mêmes, ces recherches nous ont amenés à nous poser des questions à un nouveau niveau: décrire la distribution de l'agentivité dans un réseau. (Question à laquelle nous n'avons toujours pas de réponse.)

Les nouveaux dispositifs qui allaient au-delà de l'écriture et dans lesquels les participants n'étaient pas forcement encadrés comme signataires d'une liste, par exemple, les obligeaient d'avoir à la fois des compétences communicatives et techniques. Au départ, pour être un blogueur il était nécessaire de construire un site! Évidemment, des outils ont été rapidement mis en place (comme le Blogger) pour donner un pas-à-pas et des templates, qui permettaient plus facilement de publier des contenus, changer des layouts, etc. C'était une inflexion clé pour la diffusion de la pratique des blogueurs. Mais il faut rappeler que cette "simplification" des tâches pour les blogueurs débutants était aussi de l'invisibilité des démarches techniques. Et donc pour permettre des nouvelles possibilités d'action communicationnelle pour les sujets dans la toile, c'était en quelque sorte nécessaire de créer une "boîte noire" qui jouait à son tour le rôle de médiateur.

Un deuxième volet critique de notre bilan, c'est qu'aujourd'hui, avec du recul, nous pouvons identifier un schéma analytique de tous 
nos travaux jusqu'à 2007 (et que nous semble plus courant qu'on pourrait le croire jusqu'à maintenant tout au moins dans les études du numérique au Brésil). Ce schéma analytique prend comme point de départ une révision plus ou moins détaillée des éléments techniques (ARPANET, TCP/IP, la diffusion des PCs, la vitesse des réseaux par câble puis par wifi, etc.). Ensuite, il est question de la plateforme spécifique, qui était dans les meilleurs de cas décortiqué, non pas tel un mode d'emploi, mais plutôt par les ressources mobilisées par les sujets en contraste avec les ressources qui ne l'étaient pas. Ce schéma, que nous avons cru être une pratique sociotechnique, est plutôt une démarche de recherche "socio+technique" au sens d'une juxtaposition entre les éléments. De fait, il est héritier d'un procédé qui consiste à établir un contexte objectif comme un scénario pour organiser les actions et même définir les "acteurs". C'est un procédé tout à fait semblable aux ethnographies classiques.

C'est donc un parcours analytique qui a commencé par l'introduction de la possibilité que les éléments techniques soient des médiateurs, et nous a permis de sortir des totalisations d'un domaine social déjà donné par une visée plus proche des pratiques, des actions observées sur le terrain. Ce que j'appelle ici "domaine du social" ce sont ces scénarios utilisés comme des outils pour organiser les actions des agents et pour l'élaboration des explications sur la signification ou les appropriations des techniques observées sur la toile ${ }^{8}$. Cependant, nous n'avons pas pu avancer face au dualisme implicite dans ces raisonnements entre le social et le technique; notre approche étant devenue une simple juxtaposition des éléments.

Le projet analytique que notre groupe de recherche essaie de consolider dans le domaine du numérique n'est pas l'étude du rôle des objets, ni l'usage tout court des objets, ni le rôle symbolique des objets, ce n'est donc pas une anthropologie des techniques. Ce projet est avant tout une volonté de savoir qui cherche à suivre la formation de liens (que nous ne dirons sociaux pour des raisons évoquées précédemment), des réseaux, des ensembles composites où se mêlent des éléments hétérogènes, et y forment (ou déforment) des hybrides contingents et circonstanciels. 
C'est bien l'image du cyborg que nous a proposé Donna Haraway (2000, orig. 1991) dans son "Manifeste cyborg". En fait, la question qu'elle pose dans son Manifeste est au cœur du projet que nous envisageons: où finit l'humain et où commence la machine? Ou, peut-être: où finit la machine et commence l'humain? Les questions me paraissent pouvoir faire du sens dans des situations particulières auxquelles l'enquête doit faire face. La métaphore du cyborg est un défi majeur face à l'ubiquité de la technique dans notre quotidien.

Sans pouvoir avancer plus dans les détails de ces recherches, mais encore une fois incitant sur le fait que malgré nos intentions et fondamentaux théoriques, notre volonté de savoir sociotechnique, nos récits produisaient une réduction de la complexité des séries de connexions que nous voulions décrire. C'est le paradoxe souligné par Bruno Latour (2013) dans un article qui porte un titre particulièrement adéquat pour faire la synthèse des arguments que je viens d'avancer: "Le tout est toujours plus petit que les parties". Encore une fois, j'insiste que notre insuffisance était et demeure dans les choix du terrain et de l'écriture, sur les politiques ethnographiques mises en marche dans les recherches. C'est cette "substance de l'empirisme anthropologique" (STRATHERN, 2010) de laquelle nous sommes redevables qui fait que notre attention soit captivée, capturée, par les êtres humains dans un monde complètement traversé par la technique et dans lequel les interactions sont toujours médiatisées. C'est toute la difficulté d'avoir un langage pour l'hybridisme et la distribution de l'agentivité comme l'ont pu faire les ethnologues mélanésiens et amérindiens.

\section{Notes}

1 Peut-être serait-il nécessaire de rappeler le souci de ne pas faire de la distinction entre "humains" et "non humains" une dichotomie.

2 Tous les liens seront alors des associations, social au sens latin du terme, comme l'a fait remarquer Bruno Latour (2006) dans son livre "Changer de société. Refaire de la sociologie". De telle sorte que j'aurai plutôt tendance à considérer que les expressions telles que "lien social" ou "relation social" portent une redondance, ou, comme le disait Eduardo Viveiros de Castro (2001), c'est une espèce de "pléonasme d'utilité temporaire".

3 La notion de réseau prend, à mon sens, une ampleur tout à fait particulière et doit faire objet d'une réflexion spécifique. Son usage courant et flou l'a transformée en une espèce de boîte noire de la sociologie et de l'anthropologie. Si dans les 
années 1950-60 elle avait un potentiel heuristique important lié aux ensembles de rapports/interactions dans lesquels l'idée d'appartenance n'était pas adéquate (voir les travaux de Redcliffe-Brown et de J.Barnes), il y a tout un travail à faire qui dépasse largement le propos de cette communication. Retenons au moins ici qu'il en faudrait faire état dans une révision approfondie de deux aspects: 1) la limitation de la notion de groupe et 2) la place des objets dans les interactions comme médiateurs. À propos des fondements d'une telle perspective pour la notion de réseau, il serait intéressant de revoir l'article de Michel Callon et Michel Ferrary (2006) intitulé "Les réseaux sociaux à l'aune de la théorie acteur-réseau".

4 Sans pouvoir ici avancer sur la notion de réseau, rappelons un passage de Marilyn Strathern dans son texte "Cutting the network" de 1987: "Le concept de réseau appelle une dentelle d'éléments hétérogènes qui composent des objets, des événements ou des ensembles de circonstances telles que celles-ci, unis par des interactions sociales: il est, en bref, un hybride imaginé dans l'état social étendu." (STRATHERN, 2010, p. 302)

5 Je fais référence ici à une affirmation (boutade?) de Marcel Mauss rapportée par Michel Leris dans un texte intitulé "Instructions sommaires pour les collecteurs d'objets ethnographiques" de 1931. Cette "boutade" a été rappelée par l'Editorial du dossier "Espèces d'objets" de la Revue L'Homme (n. 170, 2004), "La règle de la boîte de conserve", signé par Jean Jamin (2004, p. 8) : “Une boîte de conserve, par exemple, caractérise mieux nos sociétés que le bijou le plus somptueux ou que le timbre le plus rare. Il ne faut donc pas craindre de recueillir les choses même les plus humbles et les plus méprisées [...] En fouillant un tas d'ordures, on peut reconstituer toute la vie d'une société".

6 Ce bilan que je propose ici est aussi le résultat d'un travail de construction de l'Association Brésilienne de Chercheurs en Cyberculture (Associação Brasileira de Pesquisadores em Cibercultura, ABCiber: https://abciber.org.br), auquel nous avons participé dès sa fondation en 2007.

En passant, je rappelle que les travaux de l'ABCiber nous ont mis en étroite collaboration avec nos collègues des sciences de la communication, ce qui a fortement enrichit nos propres recherches ces dernières années.

7 C'est un sujet que nous avons étudié en détail, le débat sur l'ethnographie dans le cyberespace dans d'autres travaux (CRUZ et al., 2009; RIFIOTIS et al. 2010; RIFIOTIS et al., 201 1; RIFIOTIS, 2012).

8 La toile est ici envisagée comme une production permanente et non pas comme le réseau physique ni une totalité donnée d'avance. Comme le disait Antoine Hennion (2013, p. 25): “Il n'y a pas un programmeur qui ait créé le monde du web. Et il ne s'agit pas que de la toile, bien sûr, mais indissociablement des sites, des styles, des usages, des compétences, des formats, des ressources, tout ce qui s'est inventé l'un par l'autre, à des endroits qui ne sont jamais ceux qu'on avait prévus, des aspects les plus techniques aux formes d'expression les plus originales, à partir de cette capacité des réseaux à faire circuler les informations en quantité invraisemblable - développement qui inversement n'a eu lieu que parce qu'internet avait trouvé ses usages".

ILHA 


\section{Références}

AKRICH, Madeleine. Comment décrire les objets techniques? Techniques \& Culture, [S.l.], v. 54-55, p. 205-219, 2010 (orig. 1987).

CALLON, Michel; FERRARY Michel. Les réseaux sociaux à l'aune de la théorie de l'acteur-réseau. Sociologies Pratiques, [S.l.], v. 13, n. 2, p. 37-44, 2006.

CASTRO, Eduardo Viveiros. A propriedade do conceito. [S.l.]:

Communication présentée à l'Associação Nacional de Pós-Graduação e Pesquisa em Ciências Sociais (Anpocs), 2001.

CRUZ, Fernanda Guimarães; MÁXIMO, Maria Elisa; RIFIOTIS, Theophilos. Diálogos teórico-metodológicos entre Antropología y Comunicación en el estudio de centros públicos de acceso a Internet. In: FÉRNANDEZ, A. P.; MALDONADO, A. E. (orgs.) Metodologías Transformadoras: Tejiendo la red en comunicación, Educación, ciudadanía e Integración en América Latina. Caracas: Fondo Editorial CEPAP/UNESR, 2009, p. 147-175.

HARAWAY, Donna. Manifesto ciborgue: ciência, tecnologia e feminismosocialista no final do século XX. In: SILVA, T. T. (ed.). Antropologia do ciborgue: as vertigens do pós-humano. Belo Horizonte: Autêntica, 2000 (orig. 1991). p. 36-129.

HENNION, Antoine. Vous avez dit attachements? In: AKRICH, M. et al. (ed.). Débordements. Mélanges offerts à Michel Callon. Paris: Presses des Mines, 2010. p.179-190.

HENNION, Antoine. D'une sociologie de la médiation à une pragmatique des attachements. Sociologies, [S.l.], p. 1-32, 2013. Disponível em: http:// sociologies.revues.org/4353. Acesso em: 16 dez. 2019.

HINE, Christine. Virtual Ethnography. Londres: SAGE Publications, 2000 .

HINE, Christine. Virtual Methods: issues in social research on the internet. Londres: Berg Publishers, 2005.

JAMIN, Jean. Editorial. La règle de la boîte de conserve. Revue L'Homme (Dossier "Espèces d'objets"), n. 170, p. 7-10, 2004.

LÉVY, Pierre. A inteligência coletiva. São Paulo: Loyola, 2003.

LATOUR, Bruno. Changer de société: refaire de la sociologie. Paris: Découverte, 2006a.

LATOUR, Bruno. La science en action: introduction à la sociologie des sciences (réédition en poche). Paris: Découverte, 2006b. 
LATOUR, Bruno. Le tout est toujours plus petit que les parties: une expérimentation numérique des monades de Gabriel Tarde. Réseaux, [S.l.], v. 31, n. 177, p. 199-233, 2013.

MAUSS, Marcel. Les techniques et la technologie. Revue du MAUSS, [S.l.], n. 23, p. 434-450, 2004.

MÁXIMO, Maria Elisa; RIFIOTIS, Theophilos; SEGATA, Jean; Cruz, Fernanda Guimarães. A etnografia como método: vigilância semântica e metodológica nas pesquisas no ciberespaço. In: MALDONADO, A. E. et al. (org.). Epistemologia, investigação e formação científica em comunicação. Rio do Sul: Unidavi, 2012. p. 293-319.

QUÉRÉ, Louis. Les boîtes noires de Bruno Latour ou le lien social dans la machine. Réseaux, [S.l.], v. 7, n. 36, p. 95-117, 1989.

RIFIOTIS, Theophilos. Desafios contemporâneos para a antropologia do ciberespaço. Civitas - Revista de Ciências Sociais, Rio Grande do Sul, v. 12, n. 3, p. 566-578, 2012.

RIFIOTIS, Theophilos. Antropologia do Ciberespaço: questões teóricometodológicas sobre pesquisa de campo e modelos de sociabilidade. In: Antropologia no Ciberespaço. Florianópolis: Editora da UFSC, 2010. p. 15-27.

SEGATA, Jean; RIFIOTIS, Theophilos. (orgs.). Políticas Etnográficas no Campo da Cibercultura. Brasília: ABA Publicações, 2016.

STRATHERN, Marilyn. O Efeito Etnográfico. São Paulo: Cosac \& Naify, 2010.

VIRILO, Paul. O Espaço crítico. Rio de Janeiro: Editora 34, 1993.

Recebido em: 14/03/2018

Aceito em: 10/09/2019

\section{Theophilos Rifiotis}

Professor Titular do Departamento de Antropologia e do Programa de PósGraduação em Antropologia Social da Universidade Federal de Santa Catarina. Lecionou na Université de Montréal (Canadá) e na Universidad de Buenos Aires (Argentina) e em várias universidades no Brasil.

Endereço profissional: Universidade Federal de Santa Catarina, Centro de Filosofia e Ciências Humanas, Departamento de Antropologia. Cidade Universitária, Trindade, Florianópolis, SC. CEP: 88040-900.

E-mail: t.rifiotis@ufsc.br 